\title{
Dificultad para medir el modo, tiempo y distancia de desplazamiento en niños a actividades extraescolares. Validación cuestionario DESACEX \\ Difficulty measuring the mode, time and distance of commute in children to extracurricular activities. DESACEX questionnaire validation
}

\author{
*David Cerro-Herrero, *Mikel Vaquero-Solis, *Miguel Ángel Tapia-Serrano, *Pedro Antonio Sánchez-Miguel, **Josué \\ Prieto-Prieto \\ *Universidad de Extremadura (España), **Universidad de Salamanca (España)
}

\begin{abstract}
Resumen. Introducción: El desplazamiento activo es una oportunidad para aumentar los niveles de actividad física, es por ello que es necesario encontrar nuevos tiempos y desplazamientos habituales en los que sea interesante intervenir para aumentar los niveles de desplazamiento activo y en consecuencia de actividad física diaria. Las actividades extraescolares están en claro crecimiento ya que los padres consideran que son un elemento fundamental en la formación de sus hijos e hijas, por ello suponen un gran número de desplazamientos urbanos y de este modo se convierten en una posibilidad para fomentar el desplazamiento activo. En este sentido es necesario antes de intervenir analizar los hábitos de desplazamiento, para poder en un futuro diseñar intervenciones adecuadas. No existen instrumentos validados y por ello en la presente investigación se valida un cuestionario que sirva de herramienta para futuras investigaciones. Metodología: La muestra estuvo formada por 125 escolares de $5^{\circ}$ y $6^{\circ}$ de primaria ( 67 niños y 58 niñas), como instrumento de recogida de datos se utilizó una adaptación del cuestionario PACO para actividades extraescolares. Resultados: la mayoría de desplazamientos a las extraescolares en el estudio se realizaron en coche ( $60.3 \%$ en la ida y $59.5 \%$ en la vuelta). Los resultados muestran fiabilidad moderada en cuanto modo de desplazamiento ( $k=.62$, en la ida y $k=.50$, en el regreso) y en cuanto al tiempo que tarda en llegar $(k=.59)$ y nivel aceptable en la distancia a las extraescolares $(k=.34)$. Discusión: Los niveles de fiabilidad del cuestionario propuesto son moderados y por tanto podría ser utilizado como instrumento de medida en las variables modo de desplazamiento, acompañamiento de adultos y número de viajes semanales. Se han encontrado valores bajos de fiabilidad en las preguntas sobre distancia al centro de desarrollo de la actividad extraescolar y en la de tiempo que tarda en llegar. Por ello se han propuesto nuevas herramientas para medir dichas variables.
\end{abstract}

Palabras claves: transporte activo, actividades extraescolares, actividad física, estudiantes.

Abstract. Introduction: Active commuting is an opportunity to increase the amount of physical activity, which is why it is necessary to find new times and routines commuting in which it is interesting to intervene to increase the levels of active commuting and as a consequence, of daily physical activity. The extracurricular activities are in clear growth since the parents consider that they are a fundamental element in the formation of their sons and daughters, for that reason they suppose a great number of urban displacements and in this way they become a possibility to foment the active displacement. In this sense it is necessary before intervening to analyze the habits of displacement, to be able to design appropriate interventions in the future. There are no validated instruments and therefore, in the present investigation a questionnaire that serves as a tool for future research is validated. Methodology: The sample consisted of 125 students from the $5^{\text {th }}$ and $6 \mathrm{~h}$ degree of Primary Education ( 67 boys and 58 girls), as an instrument for data collection, an adaptation of the PACO questionnaire was used for extracurricular activities. Results: the majority of the trips to extracurricular activities/lessons in the study were by $\operatorname{car}(60.3 \%$ to the extracurricular activities and $59.5 \%$ to return home). The results showed moderate reliability in terms of travel mode $(\mathrm{K}=.62$, in the outbound and $\mathrm{K}=.50$, in the return) and in terms of the time it takes to arrive $(\mathrm{k}=0.59)$ and acceptable level in the distance to extracurricular students $(\mathrm{K}=.34)$. Discussion: The level of reliability of the proposed questionnaire is moderate and therefore, it might be used as a measuring instrument of the different types of commuting, adult accompaniment and number of weekly trips. Low values of reliability have been found in the questions about distance to the extracurricular activities school and in the time it takes to arrive. It is necessary to design new tools to measure these variables.

Keywords: active transport, extracurricular activities, physical activity, students.

\section{Introducción}

Según la Organización Mundial de la Salud (OMS) los hábitos de la mayoría de estudiantes (81\%) de entre 11 y 17 años de edad de 146 países no cumple con las recomendaciones de actividad física (Guthold, Stevens, Piley \& Bull, 2019).

Niñerola, Capdevilla y Pintanel (2006) señalaron que la actividad física ha pasado de ser un hábito integrado en las tareas diarias a considerarse una actividad de ocio y, por tanto, con una gran competencia frente a otras muchas actividades sedentarias que pueden desarrollarse en ese ámbito, como los juegos de ordenador, redes sociales, uso del teléfono móvil, películas, series de televisión, etc.

Diversas investigaciones han encontrado que un medio

Fecha recepción: 29-04-20. Fecha de aceptación: 27-08-20

David Cerro Herrero

davidcerro@unex.es para incrementar la actividad física puede ser el desplazamiento activo (Badland \& Schofield, 2008; Rojas-Reda, et al., 2016), y además aquellos estudiantes que se desplazan de forma activa suelen ser más activos que los que van en coche o transporte público al centro educativo (Berrigan et al., 2006), en este sentido el desplazamiento activo incrementa los hábitos saludables de los estudiantes.

En los últimos años se han desarrollado numerosas intervenciones para fomentar el desplazamiento activo. En el año 2011 se publicó una revisión sistemática con 14 estudios que habían desarrollado intervenciones para fomentar el desplazamiento activo y sus conclusiones mostraron que el éxito no siempre se conseguía (Chillón, Evenson, Vaughn \& Ward, 2011). Dicha revisión sistemática solo encontró efectos importantes o muy importantes en tres de las intervenciones. Posteriormente, Villa, Barranco, Evenson y Chillón (2018) actualizan la revisión del año 2011 con una revisión 23 artículos con intervenciones y cuyas conclusiones mues- 
tran un claro aumento de las investigaciones para fomentar el desplazamiento activo a los centros escolares, pero con bajos niveles de eficacia en las mejoras conseguidas con las intervenciones.

La falta de resultados en muchas intervenciones para incrementar los niveles de desplazamiento activo puede deberse al gran número de barreras existentes. Diversos estudios han denominado barreras percibidas para el desplazamiento activo a los motivos para no desplazarse de forma activa al centro educativo (Allison, Dwyer \& Makin, 1999; Silva, Vasques, Martins, Williams \& Lopes, 2011).

En estudios previos con escolares las principales barreras encontradas han sido la lejanía, el tiempo, el tráfico, la falta de aceras, el miedo, la negativa de las familias, el cansancio y la meteorología (Villa, 2011).

Ante esta problemática de falta de resultados positivos en las investigaciones sobre el desplazamiento activo en horario escolar, el presente estudio busca indagar sobre otros desplazamientos habituales de los niños y jóvenes españoles, que pueda tener menos barreras percibidas, en este sentido se ha identificado las actividades extraescolares como un contexto generador de multitud de desplazamiento y sobre el cual sería interesante indagar sobre los niveles de desplazamiento activo a estas actividades y la presencia o no de barreras para el desplazamiento activo. No se han encontrado estudios sobre los hábitos de desplazamiento a las actividades extraescolares.

Las actividades extraescolares son aquellas que se realizan fuera del horario lectivo escolar, generalmente por la tarde en aquellos colegios de jornada continua, o en las franjas horarias del mediodía o hacia la mitad de la tarde en los colegios de jornada partida. Son actividades encaminadas a potenciar otros aspectos a priori con menor tratamiento curricular en el horario lectivo y con carácter lúdico, destinadas a los distintos colectivos escolares, especialmente en la etapa de Educación Primaria (Nuñez, 2018).

El interés de los padres cada vez es mayor porque los hijos realicen un mayor número de actividades extraescolares. , Diversos estudios muestran que los resultados académicos son mejores entre los niños que realizan actividades extraescolares (Cladellas, Ramón, Clariana, Mercè, Badia, Mar \& Gotzens, 2013; Noam, Biancarosa \& Dechausay, 2002). Por otro lado, Hidalgo (2005) pone de manifiesto que no hay suficientes evidencias para afirmar el beneficio de las actividades extraescolares en la formación de los escolares de primaria. Bahamonde, Carmona, Albornoz, Hernández-García y Torres-Luque (2018) mostraron los beneficios a nivel físico de la participación en actividades extraescolares para los niños y niñas en edad escolar.

Dado el gran número de desplazamiento que generan estas actividades se plantea que podrían ser un buen espacio temporal para promover las ventajas y beneficios del desplazamiento activo, al desarrollarse además en un momento del día más cómodo para la organización y planificación familiar. De este modo poder superar las limitaciones de estudios realizados en horario escolar que no han encontrado resultados concluyentes sobre los beneficios del desplazamiento activo en horario escolar (Gelabert-Carulla, Muntaner-Mas \& Palou-Sampol, 2019) Por el horario de las familias españolas, el horario de tarde suele ser más cómodo ya que son muchas las familias donde los progenitores trabajan o estudian en horario de mañana, lo cual supone mayor complicación para organizar los desplazamientos al centro educativo al tener que combinarlos con viajes de los adultos y por tanto a pesar de que algunas familias puedan tener motivación hacia el desplazamiento activo al centro escolar, encuentran dificultades organizativas. Sin embargo, el horario de las actividades extraescolares, al ser principalmente de tarde hace que la organización familiar sea menos compleja, y por ello pueden encontrarse menos barreras para el desplazamiento activo. De este modo se hace interesante diseñar intervenciones que fomenten el desplazamiento activo a dichas actividades, pero antes de iniciar intervenciones es necesario contar con instrumentos de medida validados. Por todo ello el presente estudio tiene por objetivo desarrollar un instrumento de medida que permita medir de forma rápida los niveles de desplazamiento activo a las actividades extraescolares, para en un futuro poder plantear intervenciones que aumenten ese tipo de desplazamiento. Como objetivo secundario se plantea una aproximación al modo de desplazamiento de los escolares a las actividades extraescolares.

\section{Método}

\section{Participantes}

Los participantes en el estudio fueron un grupo de niños/as voluntarios, de diferentes colegios de la provincia de Cáceres (España), se pasó el cuestionario a un total de 125 de los cuales el 53.6\% $(n=67)$ eran chicos y el $46.4 \%(n=58)$ eran chicas. Los participantes cursaban el $43.2 \% 5^{\circ}$ de educación primaria y el $56.8 \% 6^{\circ}$ de educación primara. La muestra se seleccionó de forma aleatoria, marcando como criterios de exclusión ser menor de 10 años, con el fin de asegurar la comprensión y confiabilidad en las respuestas (Andrés, Juric \& Coni, 2010), tener alguna enfermedad o discapacidad que impida el desplazamiento activo y vivir en una localidad diferente a la del centro donde desarrollan las actividades extraescolares, ya que en este caso la elección de medio de desplazamiento está condicionada por la distancia y les será imposible optar por medios de desplazamiento activo. Una vez aplicados los criterios de exclusión fueron eliminados los datos de un sujeto por no residir en la población de su centro de actividades extraescolares y 3 por no completar el retest. Finalmente, se seleccionaron 121 (52.1\% chicos y $47.9 \%$ chicas $)$, la edad media fue de $10.79(d t=0.66)$, siendo todos los participantes estudiantes de $5^{\circ}$ primaria $(41.3 \%)$ y $6^{\circ}$ de primaria $(58.7 \%)$.

\section{Instrumento}

El cuestionario utilizado es un instrumento auto-reportado creado a partir de una adaptación del cuestionario PACO (Pedalea y Anda al Cole) de la Universidad de Granada, España (Profith, 2016), el cual ha sido utilizado en numerosas investigaciones sobre desplazamiento activo en edad escolar. Este instrumento contiene preguntas sobre el modo de desplazamiento al centro educativo y ha sido sometido a un procedimiento de validación con niños y jóvenes de entre 7 y 19 años (Chillón et al., 2017). En dicho estudio se comparó los resultados reportados por los sujetos con la medición 
objetivo del tiempo de desplazamiento a través de la aplicación Google Maps ${ }^{\mathrm{MT}}$, hallando resultados aceptables en jóvenes pero no en niños.

El presente estudio realizo una adaptación, del instrumento para poder ser utilizado en los desplazamientos a actividades extraescolares, las cuales suelen desarrollarse en horario de tarde y no siempre en el centro educativo. Para ello se creó un comité de expertos formado por cuatro investigadores en el área de educación física y deportes, con experiencia en proyectos de desplazamiento activo. Los investigadores revisaron de manera independiente la versión original y elaboraron cada uno de ellos una nueva versión adaptada al uso en actividades extraescolares. Se contrastaron las cuatro versiones y los investigadores consensuaron una versión pre definitiva.

Se realizaron entrevistas cognitivas a 12 niños y niñas de dos localidades diferentes para evaluar la comprensión, la aceptabilidad y la aplicabilidad del cuestionario; asimismo, se calculó el tiempo requerido para completarlo. Tras esta prueba piloto una nueva reunión del grupo de expertos elaboró la versión final con pequeñas modificaciones respecto a la utilizada en la prueba piloto, en cuanto a expresiones para adaptarlo a las más comunes utilizadas por los niños y niñas.

En el presente estudio se analizaron las siguientes preguntas relacionadas con el desplazamiento a las actividades extraescolares: 1) modo de desplazamiento habitual de ida, 2) modo de desplazamiento habitual de vuelta, 3) número de desplazamientos semanales, 4) distancia de desplazamiento, 5) tiempo de desplazamiento, 6) acompañamiento a la ida y 7) acompañamiento a la vuelta. En las sesiones de recogidas de datos siempre estuvo presente el mismo investigador. Las familias y los niños fueron informados sobre las características del estudio y se recogió consentimiento informado por parte de padres/madres/tutores. El estudio ha sido revisado y aceptado por el Comité ético de la Universidad de Extremadura.

\section{Procedimiento}

El grupo investigador se puso en contacto con 5 colegios para solicitar autorización, de los cuales 3 contestaron de forma positiva y de forma aleatoria se seleccionó una clase de $5^{\circ}$ o $6^{\circ}$ de primaria para desarrollar el estudio. El cuestionario fue aplicado durante los meses de abril y mayo del año 2019 en los centros educativos, a través de la metodología test-retest. De modo que se aplicó el mismo instrumento en dos momentos separados entre una y dos semanas. Para la aplicación del retest se siguieron las indicaciones de García Gutiérrez, Ruíz y Garzón (2017) de modo que se buscaron las mismas circunstancias que en el test en cuanto a día de la semana, hora, lugar de realización y profesor al cargo. La recogida de datos se desarrolló en el horario escolar, en las clases de educación física y en las horas de tutoría, se contó con la colaboración de los maestros y un investigador estuvo presente en la recogida de datos para resolver dudas y poder detectar posibles problemas de compresión del instrumento.

\section{Análisis de datos}

Para el estudio de la fiabilidad (test y retest) se calculó a través del Coeficiente de Kappa (k), Kappa Ponderado (k) y Coeficiente de Correlación de Intraclase (CCI) y su respectivo intervalo de confianza (IC). El coeficiente de Kappa es conveniente para aquellas categorías nominales las cuales no son graduales, en cambio Kappa ponderado se debe utilizar para aquellas categorías que poseen un orden de graduación. El CCI, se utiliza para valorar dos o más variables continuas y estimar el promedio de las correlaciones entre todas las posibles organizaciones de los pares de observaciones disponibles. Siguiendo en este sentido validaciones de cuestionario similares como la de Escobar, Rodríguez, Villa, Esteban y Chillón (2019). Finalmente se analizaron como variables nominales los modos de desplazamiento, la distancia, el tiempo y el acompañamiento y el número de viajes como variable continua. El análisis estadístico de los datos se realizó con el programa Statistical Package for the Social Sciences versión 12 (SPSS).

\section{Resultados}

Los estudiantes participantes en el estudio realizan la mayor parte de sus desplazamientos a las actividades extraescolares en coche $(60.3 \%$ en la ida y $59.5 \%$ a la vuelta), el siguiente modo de desplazamiento más utilizado es andar ( $37.2 \%$ de ida y un $38.0 \%$ de vuelta), siendo muy minoritarios el resto de medios de desplazamiento, en bicicleta $(1.7 \%$ tanto en la ida como en la vuelta), moto $(0.8 \%$ en la ida y $0.0 \%$ en la vuelta) y autobús público $(0.0 \%$ en la ida y $0.8 \%$ en la vuelta). Es de destacar que más de la mitad de los estudiantes va siempre acompañado ( $50.4 \%$ ida y $57.9 \%$ vuelta). Mientras que el $28.1 \%$ va a veces acompañado y el $26.4 \%$ vuelve a veces acompañado. Siendo de este modo minoritario el grupo de participantes que va de forma autónoma sin acom- 
pañamiento de adultos ( $21.5 \%$ en la ida y $15.7 \%$ en la vuelta). En cuanto a la distancia a la que viven los alumnos de las actividades extraescolares más de la mitad (54.5\%) viven a menos de 1.5 kilómetros, en concreto el $19 \%$ a menos de 0.5 kilómetros y un $35.5 \%$ entre 0.5 y 1.5 kilómetros de distancia.

Respecto al análisis de fiabilidad del test retest para el modo de desplazamiento habitual, el acompañamiento de adultos, la distancia, el tiempo en de desplazamiento y el número de desplazamiento se obtuvieron los valores de kappa: modo habitual de ida $(k=.62)$, clasificado como «moderado»; modo habitual de regreso $(k=.50)$, «moderado»; acompañamiento de adultos ida $(k=.65)$ y acompañamiento adultos vuelta $(k=.53)$, «moderado»; distancia a las extraescolares $(k=.34)$ «aceptable», tiempo que tardas en lle$\operatorname{gar}(k=.49)$, «moderado».

Respecto a las variables de viajes semanales, se obtienen valores CCI muy altos para el número de viajes andando (.86) y alto para el número de viajes en coche (.65).

La viabilidad de este cuestionario para el modo de desplazamiento fue adecuada, en su utilización con escolares no se han manifestado dificultades o dudas al responder a las preguntas. Los tiempos de respuesta de los niños/as fue entorno a los 5 minutos.

\section{Conclusiones y discusión}

El objetivo principal de este trabajo era adaptar y validar un instrumento para valorar modo habitual, tiempo y distancia de desplazamiento a las actividades extraescolares. La primera hipótesis que se formuló fue que la adaptación del cuestionario PACO a las actividades extraescolares para niños de $5^{\circ}$ y $6^{\circ}$ de primaria ( 10 a 12 años) tendría una adecuada validez y fiabilidad.

Los niveles de fiabilidad del cuestionario propuesto son moderados y por tanto podría ser utilizado como instrumento de medida en las variables modo de desplazamiento, acompañamiento de adultos y número de viajes semanales.

Se han encontrado valores bajos de fiabilidad en las preguntas sobre distancia al centro de desarrollo de la actividad extraescolar y en la de tiempo que tarda en llegar. En estudios previos se han utilizado diversidad de métodos de medición de la variable distancia al centro educativo, medición con sistema de posicionamiento global (GPS) (Duncan \& Mummery, 2007; Duncan, Mummery \& Dascombe, 2007) siendo este método de alta precisión pero de elevado coste y difícil acceso para investigadores, mapas autorreportados donde el alumno dibuja el camino real de su ruta diaria (Schantz \& Stigell,2009), cuestionarios auto administrados (Burke \& Brown, 2007; Panter, Jones, Van Sluijs \& Griffin, 2010).), Sistemas de Información Geográfica (GIS) (Timperio et al., 2006; Panter et al., 2010) y en los últimos años el uso de Google Maps se está imponiendo por ser una metodología de bajo coste y de fácil acceso a nivel mundial (Buliung, Larsen, Faulkner \& Stone, 2013). Villa, Rodríguez, Barranco, Cabezas y Chillón (2016) demostraron la alta correlación entre Google Maps y GIS, y por tanto ante el bajo coste del primero puede ser un método útil en la mayoría de investigaciones.

Como mejora al cuestionario se plantea sustituir las preguntas de a qué distancia vives del centro de extraescolares y cuánto tiempo tardas en llegar por una pregunta para identificar el centro y otra para identificar el domicilio del alumno («¿Cómo se llama el centro donde realizas tu actividad extraescolar?» $\mathrm{y}$ «¿En qué calle y número vives?») y complementar con un mapa autorreportado donde los alumnos dibujen el camino habitual que suelen utilizar.

No existen estudios para medir el nivel de desplazamiento activo actividades extraescolares a pesar del gran incremento de desplazamientos urbanos que suponen este tipo de actividades en la actualidad. Hermoso y Chinchilla (2010) analizaron los niveles de participación y satisfacción de jóvenes con actividades extraescolares y encontraron que los niveles más altos de participación se dan en la etapa de educación primaria, por lo cual es necesario adaptar los instrumentos de medida al nivel de compresión de dichas edades.

En cuanto al objetivo de hacer una primera aproximación a los hábitos de desplazamiento a las actividades extraescolares se ha observado que los escolares suelen elegir en gran medida $(54.5 \%)$ actividades que están a menos de 1.5 kilómetros de su hogar, con lo cual las posibilidades de desplazamiento activo son altas. Pero a pesar de ello son minoría los que eligen medios de desplazamiento activo, siendo el desplazamiento caminando el más habitual entre los activos y la bicicleta un medio muy residual (1.7\%). En este sentido son necesarias intervenciones para fomentar el desplazamiento activo a las actividades extraescolares, ya que el vehículo privado sigue siendo la opción prioritaria en las familias con los consiguientes riesgos medioambientales y de salud.

\section{Fortalezas y limitaciones}

El presente estudio por su novedad es una oportunidad de nuevos campos de investigación en cuanto al desplazamiento activo y la movilidad en las ciudades. En este sentido abre una nueva línea de investigación e intervención que puede permitir potenciar el desplazamiento activo en un entorno mucho más favorable que el horario lectivo, donde las familias y los escolares suelen tener mayores barreras para el desplazamiento activo.

La principal limitación es que el número de escolares con el que se ha realizado no es demasiado amplio, por lo que convendría replicar el estudio con muestras de mayor tamaño y también con diferentes rangos de tamaño de población del municipio donde se implemente.

Se plantea como mejora establecer como lugar de administración del cuestionario el centro de actividades extraescolares, para que los alumnos tengan más reciente la información sobre sus desplazamientos, aunque eso suponga tener acceso a menor muestra en cada una de las mediciones.

Como líneas futuras de investigación se planeta el diseño de estudios con participantes en extraescolares utilizando la versión propuesta del cuestionario DESACEX, y desarrollando un análisis profundo del modo del desplazamiento, así como de las distancias recorridas, nivel de acompañamiento y tiempos empleados en el desplazamiento, para a partir de los resultados iniciales poder diseñar intervenciones que mejoren los niveles de desplazamiento activo en la población infantil y juvenil y poder medir posteriormente la 
efectividad de las intervenciones diseñadas. De este modo se podrán comparar diferentes tipos de intervenciones y seleccionar aquellas más efectivas con el objetivo de mejorar los datos de desplazamiento activo en el entorno extraescolar.

\section{Referencias}

Allison, K. R., Dwyer, J. J. \& Makin, S. (1999). Perceived barriers to physical activity among high school students. Preventive medicine, 28(6), 608-615. DOI:10.1006/ pmed.1999.0489

Andrés, M. L., Juric, L. C. \& Coni, A. G. (2010). Conciencia sintáctica en niños de 5 a 8 años de edad: Diseño de un instrumento y evaluación de sus propiedades psicométricas. Avaliação Psicológica, 9(2), 199-210.

Badland, H., \& Schofield, G. (2008) Health Associations with Transport-R lated Physical Activity and Motorized Travel to Destinations. International Journal of Sustainable Transportation, 2:2, 77-90, DOI: 10.1080/ 15568310601113645

Bahamonde, C., Carmona, C., Albornoz, J., Hernández-Garcia, R. \& Torres-Luque, G. (2018). Efecto de un programa de actividades deportivas extraescolares en jóvenes chilenos (Effect of an extracurricular sport activity program on young Chileans). Retos, (35), 261-266. DOI:10.47197/ retos. v0i35.62834

Berrigan, D., Troiano, R. P., McNeel, T., DiSogra, C. \& BallardBarbash, R. (2006). Active transportation increases adherence to activity recommendations. American journal of preventive medicine, 31(3), 210-216. DOI: 10.1016/j.amepre.2006.04.007

Buliung, R. N., Larsen, K., Faulkner, G. E. \& Stone, M. R. (2013). The «path» not taken: exploring structural differences in mapped-versus shortest-network-path school travel routes. American journal of public health, 103(9), 1589-1596. DOI:10.2105/AJPH.2012.301172

Burke, M. \& Brown, A. L. (2007). Distances people walk for transport. Road \& Transport Research: A Journal of Australian and New Zealand Research and Practice, 16(3), 16.

Cladellas, R., Clariana, M., Badia, M. \& Gotzens, C. (2013). Actividades extraescolares y rendimiento académico en alumnos de primaria. European Journal of Investigation in Health, Psychology and Education, 3(2), 87-97.

Chillón, P., Ortega, F. B., Ruiz, J. R., Veidebaum, T., Oja, L., Mäestu, J. \& Sjöström, M. (2010). Active commuting to school in children and adolescents: an opportunity to increase physical activity and fitness. Scandinavian journal of public health, 38(8), 873-879.DOI:

Chillón, P., Evenson, K. R., Vaughn, A. \& Ward, D. S. (2011). A systematic review of interventions for promoting active transportation to school. International journal of behavioral nutrition and physical activity, 8(1), 10. / DOI:/10.1186/1479-5868-8-10

Chillón, P., Herrador, M., Migueles, J. H., Cabanas, V., Fernández-, J. R., Veiga, Ó. L. \& Castro-Piñero, J. (2017). Convergent validation of a questionnaire to assess the mode and frequency of commuting to and from school. Scandinavian journal of public health, 45(6), 612-620. DOI: $10.1177 / 1403494817718905$
Duncan, M. J. \& Mummery, W. K. (2007). GIS or GPS? A comparison of two methods for assessing route taken during active transport. American journal of preventive medicine, 33(1), 51-53. DOI: 10.1016/ j.amepre.2007.02.042

Duncan, M. J., Mummery, W. K. \& Dascombe, B. J. (2007). Utility of global positioning system to measure active transport in urban areas. Medicine and Science in Sports and Exercise, 39(10), 1851-1857

Escobar, D., Rodriguez, F., Villa, E., Esteban, I. \& Chillón, P. (2019). Fiabilidad y viabilidad de un cuestionario autorreportado sobre el modo, tiempo y distancia de desplazamiento en niños y adolescentes (Reliability and feasibility of a self-reported questionnaire on the mode, time and distance of commuting in children an. Retos, 37(37), 379-385

Garcia, M.P., Gutierrez, S. H., Ruíz, J. R. \& Garzón, P. C. (2017). Fiabilidad de un cuestionario de barreras del desplazamiento activo al colegio. Retos: nuevas tendencias en educación física, deporte y recreación, (32), 72-75.

Gelabert-Carulla, J., Muntaner-Mas, A. \& Palou-Sampol, P. (2019). Influencia del desplazamiento activo al colegio sobre la composición corporal y el rendimiento académico en escolares de 10-12 años (Influence of active commuting to school on body composition and academic achievement in schoolchildren aged $10-12$ years). Retos, 36(36), 376-383. DOI:10.47197/retos. v36i36.68166

Guthold, R., Stevens, G., Riley, L. \& Bull, F. (2019) Global trends in insufficient physical activity among adolescents: a pooled analysis of 298 population-based surveys with 1.6 million participants. The Lancet Child \& Adolescents Health, November 21, 2019. DOI:10.1016/ S2352-4642(19)30323-2

Hermoso, Y. \& Chinchilla, J. L. (2010). La participación en actividades extraescolares de jóvenes y adolescentes y el nivel satisfacción en la ocupación de su tiempo libre. International Journal of Developmental and Educational Psychology, 2(1).

Hidalgo, R.F. (2005). «El impacto de las actividades extraescolares en ciencia y tecnología». En A. Martín, I. Trelles y G. Zamarrón (Eds.), Universidad y Comunicación social de la ciencia (pp. 177-197). Granada: Editorial Universidad de Granada.

Noam, G. G., Biancarosa, G. \& Dechausay, N. (2002). Afterschool education: Approaches to an emerging field. Harvard Education Press.

Niñerola, J., Capdevila, L. \& Pintanel, M. (2007). Barreras percibidas y actividad física: el autoinforme de barreras para práctica de ejercicio físico. Revista de Psicología del Deporte, 15(1)

Núñez, N. L. (2018). La danza como actividad extraescolar en el alumnado de educación primaria. Sinfonía Virtual: Revista de Música Clásica y Reflexión Musical, (35), 5.

Panter, J. R., Jones, A. P., Van Sluijs, E. M. \& Griffin, S. J. (2010). Neighborhood, route, and school environments and children's active commuting. American journal of preventive medicine, 38(3), 268-278. DOI: 10.1016/ j.amepre.2009.10.040

Profith (2016). Pedalea y anda al cole. Disponible en: http:// profith.ugr.es/ 
Rojas-Rueda D, de Nazelle A, Andersen ZJ, Braun-Fahrländer C, Bruha J, Bruhova-Foltynova H, et al. (2016) Health Impacts of Active Transportation in Europe. PLoS ONE 11(3): e0149990. DOI:10.1371/journal.pone.0149990

Schantz, P. \& Stigell, E. (2009). A criterion method for measuring route distance in physically active commuting. Medicine \& Science in Sports \& Exercise, 41(2), 472478.

Silva, K. S., Vasques, D. G., Martins, C. D. O., Williams, L. A. \& Lopes, A. S. (2011). Active commuting: prevalence, barriers, and associated variables. Journal of Physical Activity and Health, 8(6), 750-757. DOI:10.1123/ jpah.8.6.750

Timperio,A., Ball, K., Salmon, J., Roberts, R., Giles-Corti, B., Simmons, D., ... \& Crawford, D. (2006). Personal, family, social, and environmental correlates of active commuting to school. American journal of preventive medicine, 30(1), 45-51. DOI: 10.1016/j.amepre.2005.08.047

Villa, E. (2011). Factores personales y ambientales asociados con el desplazamiento activo al colegio de los escolares españoles. (Proyecto fin de Máster). Universidad de Granada, España.

Villa, E., Rodríguez, C., Barranco, Y., Cabezas, L.F. \& Chillón, P. (2016). Evaluación de la concordancia de dos métodos para determinar la distancia del desplazamiento activo al colegio en escolares. Nutricion hospitalaria, 33(3), 713 71.DOI:10.20960/nh.283

Villa-Gonzalez, E., Barranco-Ruiz, Y., Evenson, K. R. \& Chillón, P. (2018). Systematic review of interventions for promoting active school transport. Preventive medicine, 111, 115-134. DOI: 10.1016/j.ypmed.2018.02.010
ANEXO: PROPUESTA FINAL DE CUESTIONARIO DESACEX

CUESTIONARIO SOBRE EL DESPLAZAMIENTO A ACTIVIDADES EXTRAESCOLARES (DESACEX) A continuación le exponemos una serie de preguntas sobre algunos aspectos relacionados con el \begin{tabular}{lrr} 
& particulares.. \\
\hline \hline Cero: Masculino & Femenino & Edad.........
\end{tabular}

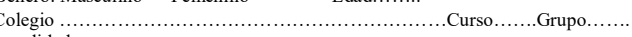

ocalidad

Nombre de la calle en la que vives

ACTIVIDADES EXTRAESCOLARES

Qué actividades extraescolares practicas: (señala todas las que realices)

Lugar (escribe el nombre

\begin{tabular}{|l|l|l|l|}
\hline Actividades motrices, fisicas o deportivas & Señala con $\mathbf{X}$ & hora & $\begin{array}{c}\text { Lugar (escrion dirección) } \\
\text { del centro y }\end{array}$ \\
\hline Actividades extraaescolares musicales & & & \\
\hline Actividades extraescolares de idiomas & & & \\
\hline Clases particulares de cualquier materia & & & \\
\hline
\end{tabular}

Cuántos días a la semana acudes a dicha actividad?: (marca con un x) 1 dial 2 dias 3 dias 4 dias 5 dias

Hora de entrada ___ ; Hora de salida

¿Cómo vas habitualmente a las Extraescolares? (Marque solo una opción. Si combina vario modos de transporte, indique aquel en el que invierta más tiempo).

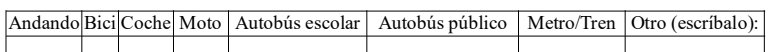

¿Cómo vuelve habitualmente su hijo/ a las Extraescolares? (Marque solo una opción. Si

combina varios modos de transporte, indique aquel en el que invierta más tiempo).

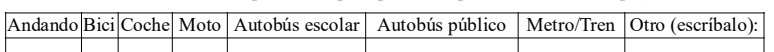

¿Vas acompañado/a de adultos a las Extraescolares

\begin{tabular}{|l|l|l|}
\hline SI & No & A veces \\
\hline
\end{tabular}

¿VUELVES acompañado/a de adultos a las Extraescolares?

\begin{tabular}{|l|l|l|}
\hline SI & No & A veces \\
\hline
\end{tabular}

¿Cuál es la dirección dónde vives?

Calle:

Dibuja en el siguiente mapa el camino que sueles realizar para llegar a las extraescolares:
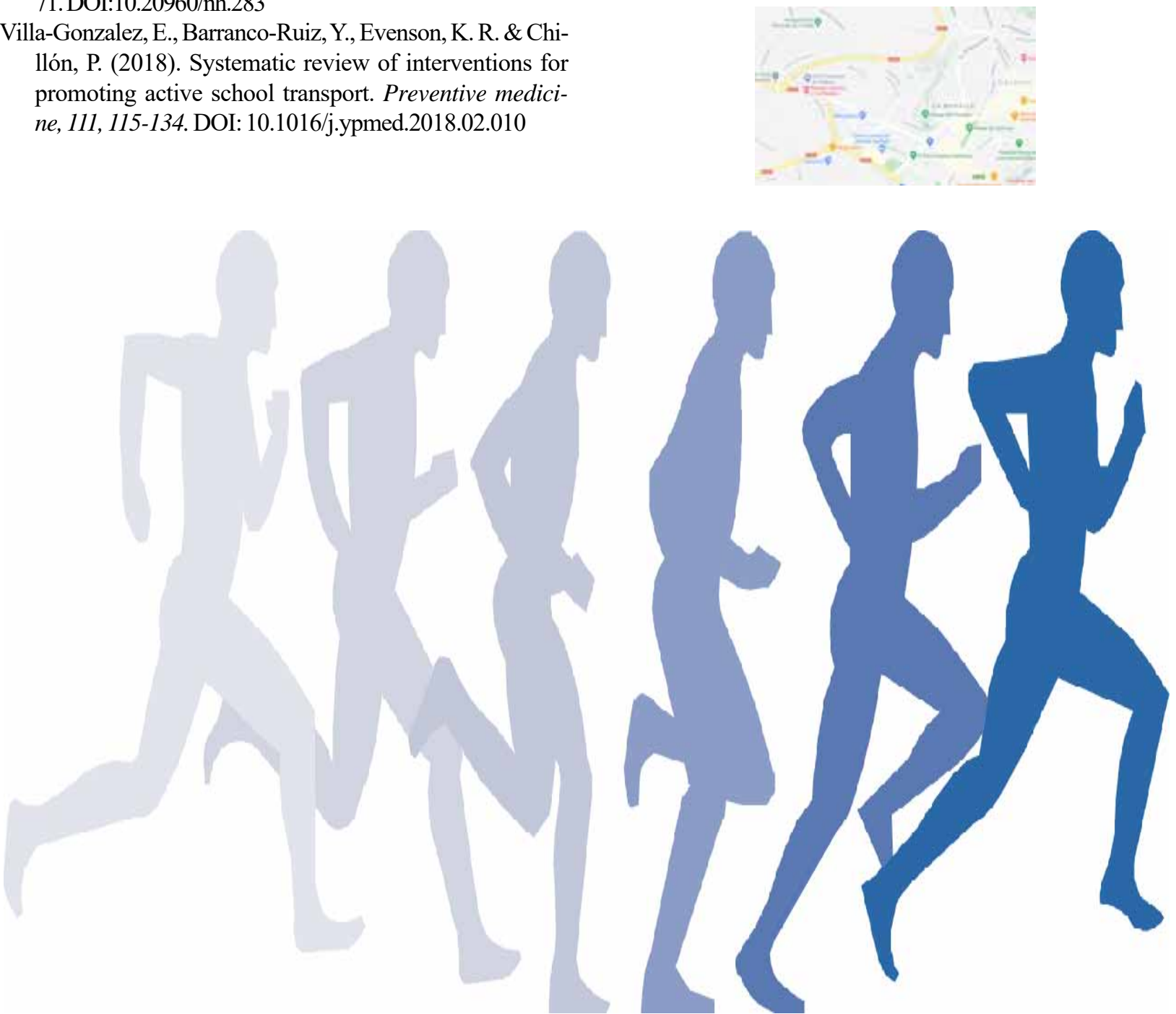